\title{
]jfis
}

\section{Application of Computational Intelligence Techniques to an Environmental Flow Formula}

Zong Woo Geem ${ }^{1}$ and Jin-Hong Kim ${ }^{2}$

${ }^{1}$ Department of Energy IT, Gachon University, Seongnam, Korea

${ }^{2}$ Department of Civil \& Environmental Engineering, Chung-Ang University, Seoul, Korea
Received: Nov. 20, 2018

Revised : Dec. 15, 2018

Accepted: Dec. 21, 2018

Correspondence to: Jin-Hong Kim

(jinhong.kim.cau@gmail.com)

(CThe Korean Institute of Intelligent Systems

cc) This is an Open Access article distributed under the terms of the Creative Commons Attribution Non-Commercial License (http://creativecommons.org/licenses/ by-nc/3.0// which permits unrestricted noncommercial use, distribution, and reproduction in any medium, provided the original work is properly cited.

\begin{abstract}
Manning formula is one of the most famous functions used in hydraulics and hydrology, which calculates the average flow velocity based on roughness coefficient, hydraulic radius, and slope. This study intends to improve the original formula by minimizing the deviation error between calculated flow velocity and observed one. The first improvement approach was to estimate the exponent values of hydraulic radius and slope, instead of using current $2 / 3$ and $1 / 2$, while fixing the roughness value. When logarithm-converted multiple linear regression, calculus-based BFGS technique, and meta-heuristic genetic algorithm were applied to the problem, genetic algorithm found the best exponent values in terms of sum of squares error and coefficient of determination. The second approach was to estimate the individual roughness value, instead of a constant one, which is the function of hydraulic radius and slope. When multiple linear regression, artificial neural network with BFGS, and artificial neural network with genetic algorithm tackled the problem, the latter found the best solution. We hope these approaches will be utilized more practically in the future.
\end{abstract}

Keywords: Computational intelligence, Manning equation, Hydraulics, Curve fitting, Genetic algorithm, Artificial neural network

\section{Introduction}

The Manning formula, also referred to as Gauckler-Manning formula (or Gauckler-ManningStrickler formula) in Europe [1] or Manning's equation in the United States [2] and Englishspeaking countries [3], is a simple-structured empirical formula which calculates the average velocity of uniform or gradually varied flow [2] in an open channel. The common form of the formula is

$$
V=\frac{a}{n} R^{2 / 3} S^{1 / 2}
$$

where $V$ is cross-sectional mean velocity $(\mathrm{L} / \mathrm{T}) ; n$ is roughness coefficient $\left(\mathrm{T} / \mathrm{L}^{1 / 3}\right) ; R$ is hydraulic radius $(\mathrm{L})$, which is calculated from the cross-sectional area $A\left(\mathrm{~L}^{2}\right)$ divided by wetted perimeter $P(\mathrm{~L})$ of the flow. For wide rectangular channels, $R$ is approximated by flow depth [1, 4]; $S$ is channel slope (L/L) which is assumed to be equal to friction slope [5]; and $a$ is conversion factor between SI $(a=1)$ and English $(a=1.49)$ units. This paper will follow the SI unit. 


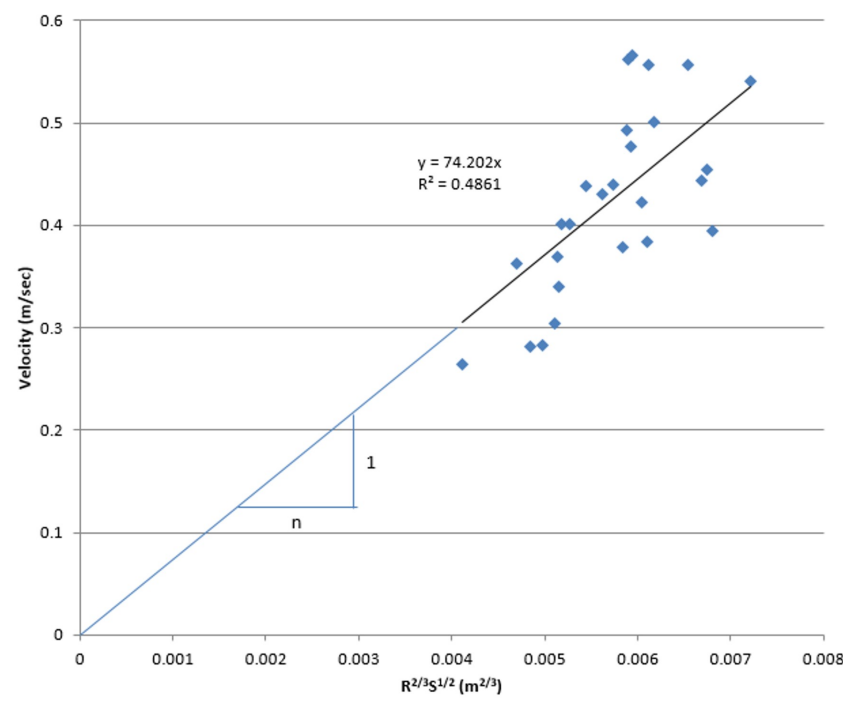

Figure 1. Relationship plot of fixed roughness coefficient.

The Manning formula is utilized for fully rough turbulent flows [1] and is usually applied to free surface profile calculation in an open channel or circular-piped sewer design [2].

This empirical formula sometimes does not work very well because it is sensitive to roughness value $n$, which is dischargedependent, and applied only to a specific flow condition (fully turbulent). The objective of this study is to improve the original Manning formula by optimally relating the calculated velocity from the formula and the observed velocity from real laboratory experiments using various statistical, optimization, and metaheuristic methods. For the dataset, Jorissen's result in 1938 [6] was used, which had been obtained after 26 runs with different conditions (observed mean velocity $=0.2640-0.5658 \mathrm{~m} / \mathrm{s}$; channel width $=0.610 \mathrm{~m}$; water depth $=0.0204-0.1049 \mathrm{~m}$; slope $=0.00111-0.00333 ;$ temperature $=18.9-23.3^{\circ} C$; bed condition = plane, ripples, or dunes) as shown in the second, third and fourth columns of Table 1. Actually, Jorissen's data can be found in Johnson's report [7] and it was again introduced by Brownlie [8].

\section{Improvement of the Formula with Fixed Rough ness Coefficient}

If the Manning's roughness coefficient $n$ is assumed to be fixed, we can rearrange Eq. (1) as follows:

$$
\frac{V}{R^{2 / 3} S^{1 / 2}}=\frac{1}{n}
$$

Also, Eq. [2] can be plotted using the dataset [8] as shown in
Figure 1.

In order to find fixed $n$, the dataset was analyzed using a linear regression with zero intercept (0I-LR; $y=c x+0)$ which passes the origin point (both $x$ and $y$ are equal to zero) and whose slope becomes $1 / n$ [9]. The regression result shows that $1 / n=74.202$ or $n=0.013477$ with the coefficient of determination $\left(R^{2}\right)$ of 0.4861 .

In this situation, is there any way to further improve the Manning formula with fixed roughness coefficient? For this "empirical" formula, the exponent values, such as $2 / 3$ of hydraulic radius and $1 / 2$ of slope, appear somewhat arbitrary and those values can be optimized using several techniques [10] such as multiple linear regression (MLR), calculus-based optimization, and meta-heuristic optimization.

For an optimal exponent estimation using MLR, we can first apply a logarithm to both sides of Eq. (1):

$$
\begin{aligned}
\ln (V) & =\ln \left(\frac{1}{n} R^{\alpha} S^{\beta}\right) \\
& =\ln \left(\frac{1}{n}\right)+\alpha \ln (R)+\beta \ln (S) .
\end{aligned}
$$

And, the common form of MLR is

$$
Y=c_{0}+c_{1} X_{1}+c_{2} X_{2} .
$$

Since we already have the dataset of $V(Y=\ln (V)), R$ $\left(X_{1}=\ln (R)\right)$, and $S\left(X_{2}=\ln (S)\right)$, the optimal values of $\alpha$, $\beta$, and $n$ can be obtained using the following relations:

$$
\alpha=c_{1}, \quad \beta=c_{2}, \quad n=1 / e^{c_{0}} .
$$

The regression result shows that $\alpha=0.785254, \beta=0.31775$, and $n=0.03037$ with $R^{2}$ of 0.8294 , which means that $\alpha$ increased from 0.6667 to $0.7853, \beta$ decreased from 0.5 to 0.3178 , $n$ increased from 0.0135 to 0.0304 (since the exponent values were changed, we might not fairly compare the magnitude of two roughness coefficients), and $R^{2}$ soared from 0.4861 to 0.8294 as shown in Figure 2.

Instead of using the above logarithm-converted multiple linear regression (Ln-MLR), we can adopt optimization techniques for estimating the roughness coefficient and two exponent values. In this optimization process, we have to minimize the sum of squares error (SSE) as follows:

$$
\text { Minimize } \sum_{i=1}^{n}\left(V_{i}-\hat{V}_{i}\right)^{2}, \quad \hat{V}_{i}=\frac{1}{n} R_{i}^{\alpha} S_{i}^{\beta},
$$


Table 1. Experimental dataset and calculated velocities with fixed $n$

\begin{tabular}{|c|c|c|c|c|c|c|c|}
\hline \multirow{2}{*}{ Run No. } & \multirow{2}{*}{$V_{i}(\mathrm{~m} / \mathrm{s})$} & \multirow{2}{*}{$R_{i}(\mathrm{~m})$} & \multirow{2}{*}{$S_{i}$} & \multicolumn{4}{|c|}{$\hat{V}_{i}(\mathrm{~m} / \mathrm{s})$} \\
\hline & & & & OI-LR & Ln-MLR & BFGS & GA \\
\hline 1 & 0.400945 & 0.060583 & 0.00113 & 0.384760 & 0.421653 & 0.427683 & 0.424328 \\
\hline 2 & 0.440086 & 0.070916 & 0.00112 & 0.425455 & 0.475809 & 0.481878 & 0.480858 \\
\hline 3 & 0.493025 & 0.073364 & 0.00113 & 0.437130 & 0.490042 & 0.495939 & 0.495704 \\
\hline 4 & 0.557127 & 0.078054 & 0.00112 & 0.453548 & 0.513028 & 0.518989 & 0.519815 \\
\hline 5 & 0.340164 & 0.044717 & 0.00167 & 0.382025 & 0.376098 & 0.377570 & 0.375687 \\
\hline 6 & 0.421810 & 0.056999 & 0.00167 & 0.449112 & 0.455053 & 0.455539 & 0.457541 \\
\hline 7 & 0.443044 & 0.066841 & 0.00165 & 0.496423 & 0.513707 & 0.513518 & 0.518728 \\
\hline 8 & 0.540074 & 0.074797 & 0.00165 & 0.535074 & 0.561139 & 0.560194 & 0.568344 \\
\hline 9 & 0.263983 & 0.019121 & 0.00332 & 0.305722 & 0.240101 & 0.237589 & 0.234723 \\
\hline 10 & 0.304127 & 0.026482 & 0.00331 & 0.379283 & 0.309773 & 0.305399 & 0.305505 \\
\hline 11 & 0.379098 & 0.032199 & 0.00333 & 0.433381 & 0.361859 & 0.355859 & 0.358765 \\
\hline 12 & 0.454098 & 0.039972 & 0.00333 & 0.500578 & 0.428822 & 0.420646 & 0.427642 \\
\hline 13 & 0.369026 & 0.060583 & 0.00111 & 0.381340 & 0.419267 & 0.425533 & 0.421913 \\
\hline 14 & 0.401014 & 0.062495 & 0.00112 & 0.391069 & 0.430846 & 0.436987 & 0.433936 \\
\hline 15 & 0.438431 & 0.065183 & 0.00113 & 0.403997 & 0.446593 & 0.452592 & 0.450315 \\
\hline 16 & 0.561799 & 0.073882 & 0.00112 & 0.437237 & 0.491367 & 0.497396 & 0.497130 \\
\hline 17 & 0.565847 & 0.074797 & 0.00112 & 0.440840 & 0.496139 & 0.502155 & 0.502126 \\
\hline 18 & 0.363013 & 0.038834 & 0.00168 & 0.348775 & 0.337300 & 0.339108 & 0.335656 \\
\hline 19 & 0.430440 & 0.051457 & 0.00165 & 0.416984 & 0.418323 & 0.419451 & 0.419442 \\
\hline 20 & 0.477283 & 0.055201 & 0.00167 & 0.439616 & 0.443742 & 0.444382 & 0.445783 \\
\hline 21 & 0.500336 & 0.059032 & 0.00166 & 0.458349 & 0.466857 & 0.467263 & 0.469848 \\
\hline 22 & 0.556912 & 0.064065 & 0.00167 & 0.485498 & 0.498785 & 0.498636 & 0.503096 \\
\hline 23 & 0.282029 & 0.024382 & 0.00333 & 0.360036 & 0.290865 & 0.286975 & 0.286223 \\
\hline 24 & 0.283186 & 0.025394 & 0.00332 & 0.369376 & 0.300019 & 0.295897 & 0.295552 \\
\hline 25 & 0.383270 & 0.034578 & 0.00331 & 0.453106 & 0.381960 & 0.375394 & 0.379418 \\
\hline 26 & 0.394911 & 0.040649 & 0.00332 & 0.505460 & 0.434107 & 0.425792 & 0.433106 \\
\hline
\end{tabular}

where $V_{i}$ is $i$ th observed mean velocity in dataset; $\hat{V}_{i}$ is calculated mean velocity using $i$ th hydraulic radius $R_{i}$ and slope $S_{i}$ in the dataset.

When a calculus-based optimization technique, named BFGS [11], was applied to the identical dataset, we could obtain $\alpha=$ $0.773554, \beta=0.282297$, and $n=0.03936$ with $R^{2}$ of 0.8295 and SSE of 0.0345 , which means that $R^{2}$ was slightly improved from 0.8294 to 0.8295 and SSE was also slightly improved from 0.0347 to 0.0345 when compared with the results from Ln-MLR.

When a popular meta-heuristic algorithm, named genetic algorithm (GA) [12], was also applied to the identical dataset, we could obtain $\alpha=0.812227, \beta=0.319725$, and $n=$
0.027611 with $R^{2}$ of 0.8309 and SSE of 0.0342 , which means that $R^{2}$ was even improved from 0.8295 to 0.8309 and SSE was also even improved from 0.0345 to 0.0342 when compared with the results from BFGS. The fifth to eighth columns of Table 1 show the calculated velocities from different techniques, and Table 2 summarizes corresponding parameter values and fitness indexes.

\section{Improvement of the Formula with Varied Rough- ness Coefficient}

Roughness value $n$ varies along the reach in a river, and even varies in an identical reach with different flow amount [4]. The 


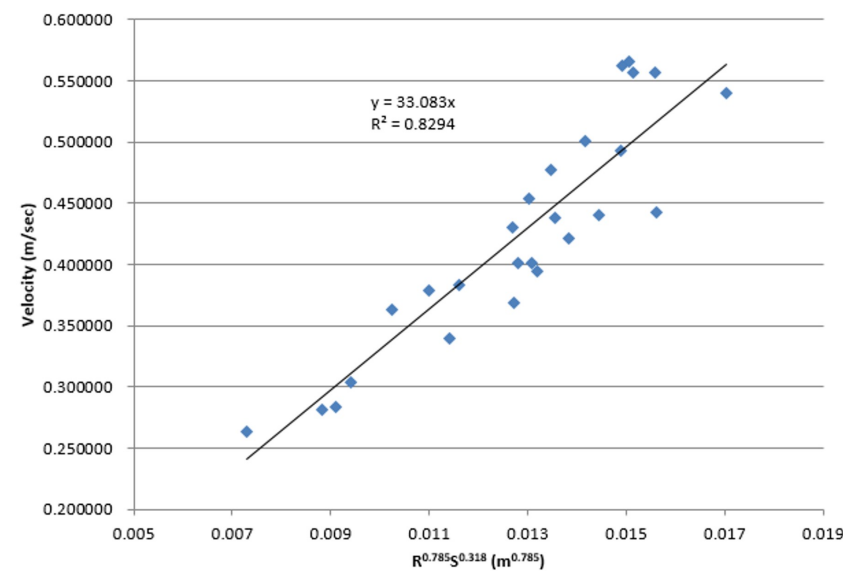

Figure 2. Relationship plot using multiple linear regression.

Table 2. Optimal parameter values from various techniques

\begin{tabular}{cccccc}
\hline Method & $\alpha$ & $\beta$ & $n$ & $R^{2}$ & SSE \\
\hline OI-LR & 0.6667 & 0.5000 & 0.0135 & 0.4861 & 0.1041 \\
Ln-MLR & 0.7853 & 0.3178 & 0.0304 & 0.8294 & 0.0347 \\
BFGS & 0.7736 & 0.2823 & 0.0394 & 0.8295 & 0.0345 \\
GA & 0.8122 & 0.3197 & 0.0276 & 0.8309 & 0.0342 \\
\hline
\end{tabular}

value of $n$ generally decreases as discharge (or depth or hydraulic radius) increases [13-15] although sometimes a direct proportion between the two occurs in sand-bed, instead of this inverse proportion [3, 14]. Anyhow, this discharge-dependency is seldom considered, and most cases adopt a fixed roughness value [3] for a given reach.

If the Manning's roughness coefficient $n$ is assumed to be varied, we can calculate individual roughness coefficient as follows:

$$
n_{i}=\frac{R^{2 / 3} S^{1 / 2}}{V_{i}}
$$

The individual roughness value is shown in the third column of Table 3 . When a graph of $R_{i}$ versus $n_{i}$ is drawn as shown in Figure 3 , we can confirm the previous studies where roughness decreases with increasing hydraulic radius.

Furthermore, when a graph of $S_{i}$ versus $n_{i}$ is drawn as shown in Figure 4, we can also confirm the previous research where roughness increases with increasing slope [16].

With the above relationships among hydraulic radius, slope and roughness, we can derive a two-independent-variable func-

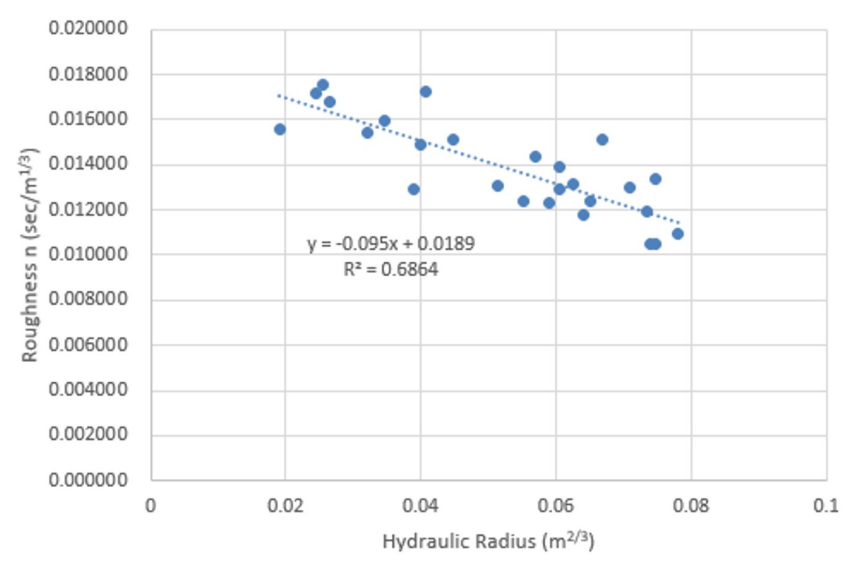

Figure 3. Relationship plot between hydraulic radius and roughness.

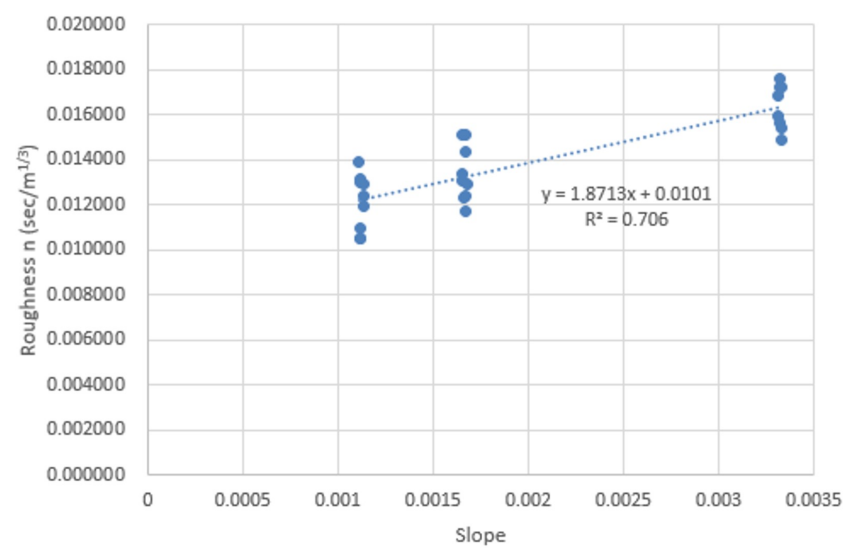

Figure 4. Relationship plot between slope and roughness.

tion using MLR as follows:

$$
n_{i}=c_{0}+c_{1} R_{i}+c_{2} S_{i}
$$

MLR could obtain $c_{0}$ of $0.014049, c_{1}$ of -0.04483 , and $c_{0}$ of 1.09974 with $R^{2}$ of 0.8367 and SSE of 0.0333 . The corresponding $n_{i}$ and $\hat{V}_{i}$ values can be found in the seventh and fourth columns of Table 3, and Figure 5 shows the relationship between observed and calculated velocities. This varied roughness approach by MLR slightly enhanced the solution quality in terms of $R^{2}$ from 0.8309 to 0.8367 , and SSE from 0.0342 to 0.0333 when compared with those from the fixed roughness approach by GA.

In the varied roughness approach by MLR, the roughness $n_{i}$ was the function of hydraulic radius $R_{i}$ and slope $S_{i}$. We can ask if there is any way to improve this linear relationship among them.

This study introduces an artificial neural network (ANN) 
Table 3. Experimental dataset and calculated velocities with varied $n$

\begin{tabular}{|c|c|c|c|c|c|c|c|c|}
\hline \multirow{2}{*}{ Run Ne } & \multirow{2}{*}{$V_{i}(\mathrm{~m} / \mathrm{s})$} & \multirow{2}{*}{$n_{i}\left(\mathrm{~s} / \mathrm{m}^{1 / 3}\right)$} & \multicolumn{3}{|c|}{$\hat{V}_{i}(\mathrm{~m} / \mathrm{s})$} & \multicolumn{3}{|c|}{$\hat{n}_{i}$} \\
\hline & & & MLR & $\begin{array}{l}\text { ANN+ } \\
\text { BFGS }\end{array}$ & $\begin{array}{c}\text { ANN+ } \\
\text { GA }\end{array}$ & MLR & $\begin{array}{l}\text { ANN+ } \\
\text { BFGS }\end{array}$ & $\begin{array}{c}\text { ANN+ } \\
\text { GA }\end{array}$ \\
\hline 1 & 0.400945 & 0.012933 & 0.412319 & 0.418819 & 0.410950 & 0.012576 & 0.012381 & 0.012618 \\
\hline 2 & 0.440086 & 0.013029 & 0.473793 & 0.487133 & 0.471736 & 0.012102 & 0.011770 & 0.012155 \\
\hline 3 & 0.493025 & 0.011949 & 0.490799 & 0.505732 & 0.496809 & 0.012003 & 0.011649 & 0.011858 \\
\hline 4 & 0.557127 & 0.010971 & 0.518795 & 0.538541 & 0.585896 & 0.011782 & 0.011350 & 0.010432 \\
\hline 5 & 0.340164 & 0.015135 & 0.370899 & 0.364315 & 0.379831 & 0.013881 & 0.014132 & 0.013555 \\
\hline 6 & 0.421810 & 0.014349 & 0.454040 & 0.445487 & 0.453332 & 0.013330 & 0.013586 & 0.013351 \\
\hline 7 & 0.443044 & 0.015100 & 0.519934 & 0.512035 & 0.509077 & 0.012867 & 0.013066 & 0.013142 \\
\hline 8 & 0.540074 & 0.013352 & 0.576392 & 0.570223 & 0.560367 & 0.012511 & 0.012646 & 0.012868 \\
\hline 9 & 0.263983 & 0.015608 & 0.244620 & 0.252857 & 0.254519 & 0.016843 & 0.016294 & 0.016188 \\
\hline 10 & 0.304127 & 0.016807 & 0.309751 & 0.315694 & 0.316054 & 0.016502 & 0.016191 & 0.016173 \\
\hline 11 & 0.379098 & 0.015406 & 0.359028 & 0.362360 & 0.361278 & 0.016268 & 0.016118 & 0.016166 \\
\hline 12 & 0.454098 & 0.014856 & 0.423771 & 0.421844 & 0.417734 & 0.015919 & 0.015992 & 0.016149 \\
\hline 13 & 0.369026 & 0.013926 & 0.409370 & 0.416470 & 0.407822 & 0.012554 & 0.012340 & 0.012602 \\
\hline 14 & 0.401014 & 0.013143 & 0.422327 & 0.430116 & 0.419148 & 0.012479 & 0.012253 & 0.012574 \\
\hline 15 & 0.438431 & 0.012418 & 0.440150 & 0.449151 & 0.435160 & 0.012370 & 0.012122 & 0.012512 \\
\hline 16 & 0.561799 & 0.010489 & 0.492323 & 0.508122 & 0.502058 & 0.011969 & 0.011597 & 0.011737 \\
\hline 17 & 0.565847 & 0.010499 & 0.498087 & 0.514702 & 0.514645 & 0.011928 & 0.011543 & 0.011544 \\
\hline 18 & 0.363013 & 0.012948 & 0.332046 & 0.326778 & 0.343594 & 0.014156 & 0.014384 & 0.013680 \\
\hline 19 & 0.430440 & 0.013055 & 0.414518 & 0.406985 & 0.419285 & 0.013557 & 0.013808 & 0.013403 \\
\hline 20 & 0.477283 & 0.012413 & 0.441769 & 0.433391 & 0.442782 & 0.013411 & 0.013670 & 0.013380 \\
\hline 21 & 0.500336 & 0.012346 & 0.466955 & 0.458501 & 0.464440 & 0.013228 & 0.013472 & 0.013300 \\
\hline 22 & 0.556912 & 0.011749 & 0.502771 & 0.494049 & 0.494495 & 0.013014 & 0.013243 & 0.013232 \\
\hline 23 & 0.282029 & 0.017204 & 0.291977 & 0.298950 & 0.299863 & 0.016618 & 0.016231 & 0.016181 \\
\hline 24 & 0.283186 & 0.017578 & 0.300571 & 0.307062 & 0.307717 & 0.016562 & 0.016212 & 0.016177 \\
\hline 25 & 0.383270 & 0.015932 & 0.378361 & 0.380001 & 0.377960 & 0.016139 & 0.016069 & 0.016156 \\
\hline 26 & 0.394911 & 0.017249 & 0.429020 & 0.426441 & 0.421927 & 0.015878 & 0.015974 & 0.016145 \\
\hline
\end{tabular}

approach with optimization technique, which can consider nonlinearity among variables. Actually ANN approaches have been applied to various prediction problems such as energy demand [17] or water pipe deterioration [18]. While those approaches used error-back-propagation technique to adjust the weight values among layers, a recent ANN approach hybridized ANN with an optimization algorithm to search the solution space of an ocean engineering problem more efficiently [19]. The basic ANN model, named feed-forward multilayer perceptron, can be structured as shown in Figure 6. As shown in the figure, there are three layers (input layer in left column, hidden layer in middle column, and output layer in right column). If we put certain values for hydraulic radius
$R_{i}$ and slope $S_{i}$ in the left input layer, these values are multiplied by weighting values (such as 16.18) on the links. Then, those multiplied values are summed at each node (for example, $\left.v_{1}=16.18 \times R_{i}+(-9.36) \times S_{i}+(-18.21)\right)$ in the middle hidden layer. Again, Sigmoid-functioned nodal values (for example, $\frac{1}{1+e^{-v_{1}}}$ ) in the middle layer are multiplied by weighting values (such as -97.43 ), then the multiplied values are finally summed (for example, $z=\frac{-97.43}{1+e^{-v_{1}}}+\frac{-1.65}{1+e^{-v_{2}}}+2.52$ ) in the right output layer and Sigmoid functioned.

When the ANN was hybridized with BFGS (ANN+BFGS), this model slightly enhanced the solution quality when compared with MLR, obtaining $R^{2}$ of 0.8447 and SSE of 0.0315 . The corresponding $n_{i}$ and $\hat{V}_{i}$ values can be found in the eighth 


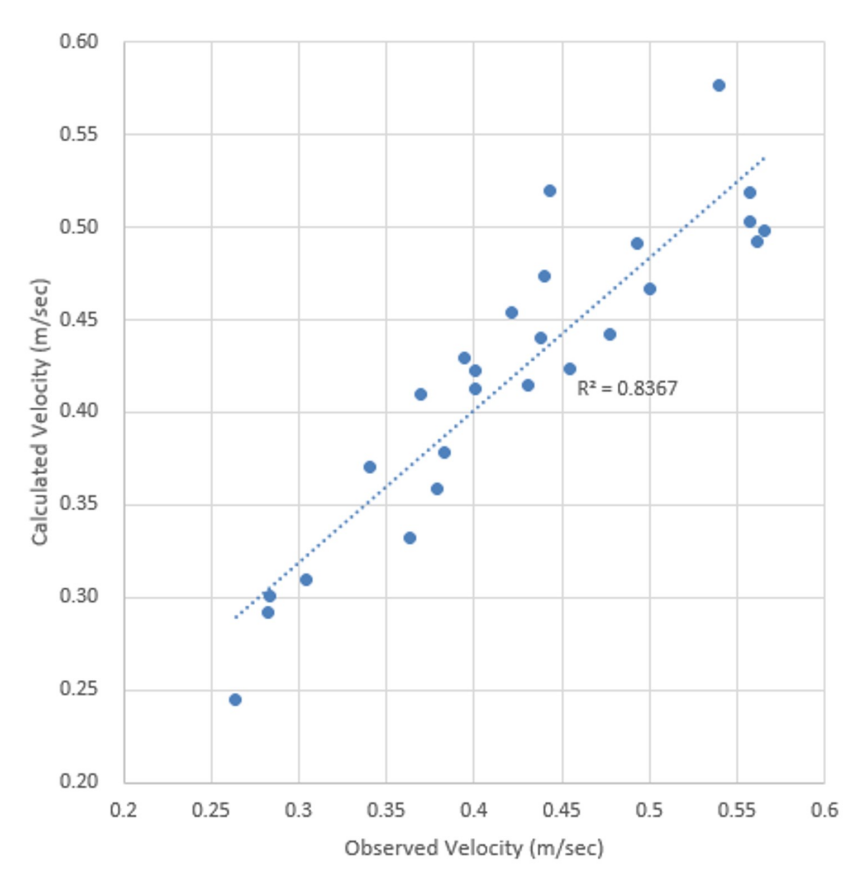

Figure 5. Observed and calculated velocities from MLR.

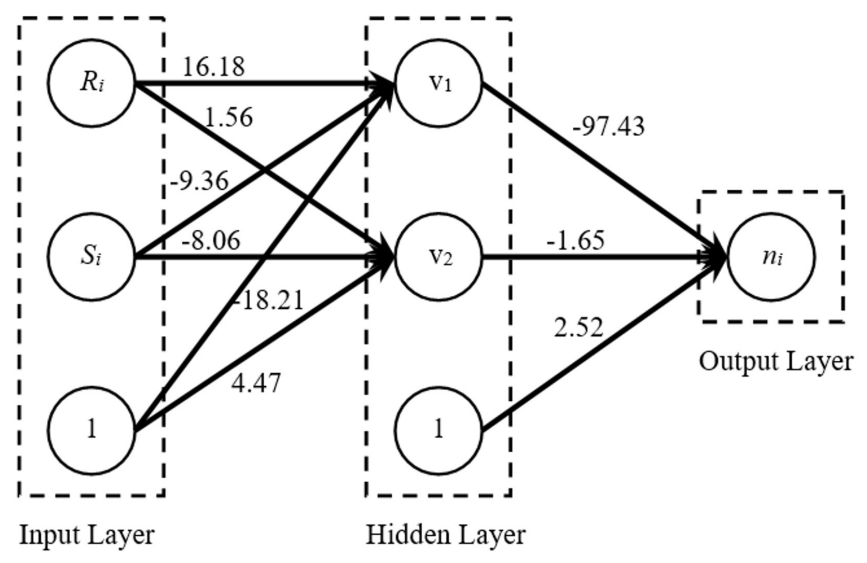

Figure 6. Structure of feed-forward multilayer perceptron model.

and fifth columns of Table 3 .

When the ANN was hybridized with GA (ANN+GA), this model even enhanced the solution quality when compared with ANN+BFGS, obtaining $R^{2}$ of 0.8636 and SSE of 0.0277. The corresponding $n_{i}$ and $\hat{V}_{i}$ values can be found in the ninth and sixth columns of Table 3. The optimal weight values from ANN+GA are shown in Figure 6, and this result can be explicitly described as follows:

$$
\begin{aligned}
& \hat{n}_{i}=\frac{0.0176}{1+e^{-z}} \\
& z=\frac{-97.43}{1+e^{-v_{1}}}+\frac{-1.65}{1+e^{-v_{2}}}+2.52
\end{aligned}
$$

$$
\begin{aligned}
& v_{1}=\frac{R_{i}}{0.0781} \times 16.18+\frac{S_{i}}{0.0033} \times(-9.36)-18.21, \\
& v_{2}=\frac{R_{i}}{0.0781} \times 1.56+\frac{S_{i}}{0.0033} \times(-8.06)+4.47 .
\end{aligned}
$$

\section{Discussions}

The amount of data (26 points) introduced in Table 1 does not appear very large. Nonetheless, this is not small for our regression-type neural-network calculation. In fact, a much larger dataset is required for unstructured data (pixel-type photos or sound wave files). However, because our hydraulic data is very structured (number-type), 26 points might be enough for devising the better formula, which could be verified with statistical indexes ( $R^{2}$ and SSE) and our other papers for energy demand prediction [17] and pipe condition assessment [18].

Actually, Manning formula can be derived from Chézy formula [13] as follows:

$$
V=C R^{1 / 2} S^{1 / 2}
$$

where $C$ is Chézy number and can be represented as $C=$ $R^{1 / 6} n^{-1}$. This is why the Manning formula is sometimes called as Chézy-Manning formula [20].

Since the basic structure of the Chézy formula relies on a physical relationship between the average velocity and the square root of the bed shear stress $(\tau \propto R S)$, we may change only Chézy number while fixing the exponent 0.5 . When BFGS tackled this situation (fixing $R^{1 / 2} S^{1 / 2}$ part of Chézy formula), we obtained a much higher exponent value (1.0024) for $R$ in Chézy number with $R^{2}=0.8075$ and $\mathrm{SSE}=0.0412$, which is a more theoretical approach but less accurate when compared with the approach of varied slope exponent.

Manning formula is usually used by engineers, who barely have much knowledge of cutting-edge computational intelligence techniques, such as artificial neural network and genetic algorithm. The ANN model in this study can be simply an improved model which considers higher nonlinearity between independent variables and a dependent one, than existing MLR model. And, the GA technique can be utilized for globally finding optimal weighting values of the ANN model. However, a calculus-based BFGS model, which finds optimal weighting values locally, could perform as well as GA in this study. In order for engineers to easily use these techniques, application software can be coded in the form of spreadsheet macros. 


\section{Conclusions}

This study proposed various techniques to improve the original Manning formula. The approach was divided into two parts. The first approach was to estimate the exponents of hydraulic radius and slope while fixing the roughness value. When three different techniques, such as logarithm-converted MLR, gradient-based BFGS, and meta-heuristic GA as well as zero-intercept LR, tackled the problem, the GA technique found the best solutions with respect to determination coefficient and SSE.

The second approach was to estimate the individual roughness value while fixing the exponent values of hydraulic radius and slope. When three different techniques, such as MLR, $\mathrm{ANN}+\mathrm{BFGS}$, and ANN+GA, tackled the problem, ANN+GA found the best solutions with respect to determination coefficient and SSE.

Nonetheless, we have to admit that this study has a limitation when determining the Manning's roughness $n$, which is also related with riverbed material, particle size distribution, and sediment transport, mainly because we do not currently have those detailed data. However, in order to fully investigate the roughness, we need such factors as riverbed type, flow depth, hydraulic radius, free surface width, $d_{50}, d_{75}, d_{84}, d_{90}$, cross section geometry, etc, as shown in previous studies [21]. While the goal of this paper to minimize any error between experimental data and corresponding hydraulic formula is accomplished by utilizing computational intelligence techniques, we have to consider more roughness-related data for implementing more practical and realistic formula in the future. Also, various soft computing techniques such as neural network [22], meta-heuristics [23], and fuzzy theory [24] will be utilized for this endeavor.

\section{Conflicts of Interest}

The authors declare no conflict of interest.

\section{Acknowledgments}

This research was supported by a grant (12-TI-C02) from Advanced Water Management Research Program funded by Ministry of Land, Infrastructure and Transport of Korean government.

\section{References}

[1] H. Chanson, The Hydraulics of Open Channel Flow: An Introduction. Oxford, UK: Elsevier, 2004.

[2] R. Czachorski, "Manning's equation the details behind this highly versatile formula," 2018, http://www. h2ometrics.com/manning-equation/

[3] R. Ferguson, "Time to abandon the manning equation?," Earth Surface Processes and Landforms, vol. 35, no. 15, pp. 1873-1876, 2010. https://doi.org/10.1002/esp.2091

[4] Wikipedia, "Manning formula," 2018, https://en. wikipedia.org/wiki/Manningformula

[5] L. W. Mays, Water Resources Engineering. Hoboken, NJ: John Wiley \& Sons, 2005.

[6] A. L. Jorissen, "Etude experimentale du transport solide des cours d'Eau (Experimental study of the solid transport of watercourses)," Revue Universelle des Mines (Universal Journal of Mines), vol. 14, 269-282, 1938.

[7] J. W. Johnson, Laboratory Investigations on Bed-Load Transportation and Bed Roughness (SCS-TP-50). Washington, DC: U.S. Soil Conservation Service, 1943.

[8] W. R. Brownlie, "Compilation of alluvial channel data: laboratory and field," California Institute of Technology, Pasadena, CA, USA, Report No. KH-R-43B, 1981.

[9] J. S. Kim, "A study on the optimum parameter estimation of mean velocity equation," M.S. thesis, Pusan National University, Busan, Korea, 2012.

[10] Z. W. Geem, J. H. Kim, and J. O. Kim, "Improvement of flow velocity formula for nature-like fishways," African Journal of Agricultural Research, vol. 6, no. 32, pp. 66096612, 2011. https://doi.org/10.5897/AJARX11.032

[11] Z. W. Geem, "Parameter estimation for the nonlinear Muskingum model using BFGS technique," Journal of Irrigation and Drainage Engineering, vol. 132, no. 5, pp. 474-478, 2006. https://doi.org/10.1061/(ASCE)07339437(2006)132:5(474)

[12] S. Mohan, "Parameter estimation of nonlinear Muskingum models using genetic algorithm," Journal of Hydraulic Engineering, vol. 123, no. 2, pp. 137-142, 1997. https: //doi.org/10.1061/(ASCE)0733-9429(1997)123:2(137) 
[13] G. M. Hornberger, P. L. Wiberg, J. P. Raffensperger, and K. N. Eshleman, Elements of Physical Hydrology. Baltimore, MD: The Johns Hopkins University Press, 1998.

[14] H. S. Woo, River Hydraulics. Seoul, Korea: Cheongmungak, 2001.

[15] W. Kim, Y. S. Kim, and H. S. Woo, "Estimation of channel roughness coefficients in the Han river using unsteady flow model," Journal of Korean Water Resources Association, vol. 28, no. 6, pp. 133-146, 1995.

[16] M. W. Jeon, H. S. Lee, S. U. Ahn, Y. S. Cho, and M. W. Jeon, "Estimation of average roughness coefficients of Bocheng stream basin," in Proceedings of the Conference of the Korean Water Resources Association, Yongpyong, Korea, 2009, pp. 1306-1310.

[17] Z. W. Geem and W. E. Roper, "Energy demand estimation of South Korea using artificial neural network," Energy Policy, vol. 37, no. 10, pp. 4049-4054, 2009. https://doi. org/10.1016/j.enpol.2009.04.049

[18] Z.W. Geem, C. L. Tseng, J. Kim, and C. Bae, "Trenchless water pipe condition assessment using artificial neural network," in Proceedings of the International Pipeline Conference (Pipelines 2007), Boston, MA, 2007, pp. 1-9. https://doi.org/10.1061/40934(252)26

[19] A. Lee, Z. W. Geem, and K. D. Suh, "Determination of optimal initial weights of an artificial neural network by using the harmony search algorithm: application to breakwater armor stones," Applied Sciences, vol. 6, no. 6, p. 164, 2006. https://doi.org/10.3390/app6060164

[20] W. G. Strupczewski, "Warning of application of the ChezyManning formula regardless of channel shape," in Proceedings of the International Conference on Hydrology and Water Resources, New Delhi, India, 1993, pp. 371383. https://doi.org/10.1007/978-94-011-0389-3_24

[21] J. S. Lee, J. E. Oh, Y. J. Park, and J. H. Jung, "Determination of relationships for manning roughness coefficient for hydraulic engineering field," Journal of the Korean Society of Hazard Mitigation, vol. 12, no. 1, pp. 167-177, 2012. https://doi.org/10.9798/KOSHAM.2012.12.1.167
[22] H.-W. Lee, N.-R. Kim, and J.-H. Lee, "Deep neural network self-training based on unsupervised learning and dropout," International Journal of Fuzzy Logic and Intelligent Systems, vol. 17, no. 1, pp. 1-9, 2017. https: //doi.org/10.5391/IJFIS.2017.17.1.1

[23] J. H. Park, J. S. Yu, and Z. W. Geem, "Genetic algorithmbased optimal investment scheduling for public rental housing projects in South Korea,' International Journal of Fuzzy Logic and Intelligent Systems, vol. 18, no. 2, pp. 135-145, 2018. https://doi.org/10.5391/IJFIS.2018.18.2. 135

[24] W. J. Lee, H. Y. Jung, J. H. Yoon, and S. H. Choi, "Analysis of variance for fuzzy data based on permutation method," International Journal of Fuzzy Logic and Intelligent Systems, vol. 17, no. 1, pp. 43-50, 2017. https://doi.org/10.5391/IJFIS.2017.17.1.43

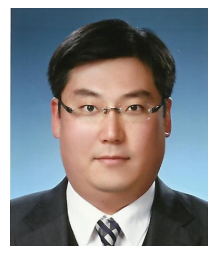

Zong Woo Geem is an associate professor in College of Information Technology, Gachon University. He received his B.E. from ChungAng University, M.Sc. from Johns Hopkins University, and Ph.D. from Korea University. He has researched at Virginia Tech, University of Maryland College Park, and Johns Hopkins University. He invented a music-inspired algorithm, Harmony Search, which has been applied to various optimization problems. His research interest includes phenomenon-mimicking algorithm and its applications to energy, environment and water fields.

E-mail: zwgeem@gmail.com

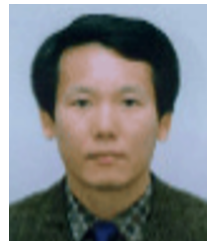

Jin-Hong Kim is a professor in Department of Civil, Environmental \& Plant Engineering, Chung-Ang University. He received his B.E. and M.Eng. from Seoul National University, and Ph.D. from University of Tokyo. His research interest includes hazard mitigation in urban flood, ecological enhancement using fish ladder, riparian vegetation behavior against flood flow, and close-to-nature river restoration.

E-mail: jinhong.kim.cau@gmail.com 